Bee XML Journal

\title{
Sharing of Data on Bees and Beekeeping
}

\author{
Walter Haefecker ${ }^{1}$ \\ ${ }^{1}$ European Professional Beekeepers Association
}

License: $\underline{\text { Creative Commons Attribution 4.0 International License (CC-BY 4.0). }}$ 


\section{Article Introduction from the Current BeeXML Chair Joseph Cazier}

On October 3, 2017, Walter Haefeker proposed the formation of Apimondia Working Group \#15 for the "Standardization of Data on Bees and Beekeeping", which was approved a few days later during the Apimondia World Congress in Istanbul, Turkey. He was joined by founding members Fani Hatijina, Joseph Cazier, James Wilkes, Norberto Garcia, Huw Evans, Agnes Rotais and Riccardo Jannoni and with the support of Etienne Bruneau, Apimondia Vice President for Beekeeping Technology and Quality. The express mission of the group is to "Enable Bee Data Sharing" through data standardization, data collection and documentation, data harmonization and by making data and policy recommendations". This journal serves as a venue for this group to carry out and communicate this work. In this article, we hear from our group's founder, Walter Haefeker, in a letter intended to set the tone for this journal.

Keywords: BeeXML, Hive Management, Hive Tracking, Apiary Management, Privacy

\section{BeeXML Introduction Letter by the Group Founder Walter Haefeker}

The amount of human- and machine-generated data is growing exponentially and the beekeeping sector is no exception. Many beekeepers are adopting digital tools to manage their hives and are adding sensors such as hive scales to remotely monitor the status of their colonies. Professional beekeepers generate both hive management and business data. Scientists have often pioneered the use of digital tools in their bee research projects. Governments are starting to add digitalization to the required reporting of colony numbers and relevant bee diseases.

Reports of declining bee health have created a heightened sense of urgency to share bee related data to better understand the multitude of factors impacting bees. The potential to use "Big Data" tools to transform all these different data sources into actionable information remains unrealized not only because of the obvious lack of standardization, but also because of missing protections for personal and business data.

Standardization appears to be the most straightforward part of this process. It involves taking an inventory of the existing data islands, finding commonalities and proposing standard representations with expanding coverage. Because of the current focus on bee health, the standardization efforts have started with colony data. However, there 
are a lot more domains in the beekeeping sector where data exist and standardization is needed.

There are not only environmental factors, but also products beekeepers use as winter feed and pollen supplements, where data on quantity and composition exist and sharing would be useful for scientific research as well as for organic certification.

A lot of information about bee genetics is also being recorded in queen breeding programs. Maintaining the data in a standardized format would not only facilitate the sharing of information among breeders, but also preserving the records, which often are maintained by a single volunteer. Just like in scientific projects, there is a big problem with orphan data in proprietary formats. Having records in a consistent format over long time periods is of increasing importance for understanding biodiversity trends and the effects of climate change on pollinators.

As bee diseases, pathogens and parasites spread geographically, standardized records on different strains as well as treatments and their effectiveness can help veterinary services develop and implement effective measures.

There are many projects monitoring environmental data as well as contaminants and residues in bee products. The results are difficult to compare because of a lack of standardization of both data and collection methods. The global COLOSS project has developed the "Bee Book" to harmonize the methods. This effort would benefit greatly, if each described method also had an associated data standard.

The beekeeping sector is not just important for pollination, but also as a source of bee products as food or for apitherapy. The data on the authenticity of bee products (NMR method etc.) as well as

market data (prices, trade flows) are an essential tool to combat fraud in the supply chain and should be maintained in a standard format globally.

But even if the data format is well defined the information may not be reliable for a whole host of reasons. Since tracking hives and hive products is essential to any wellrun beekeeping operation there is a strong incentive for beekeepers to keep accurate and current data. It does not make sense for the beekeeper to think otherwise. But if third parties like tax authorities also demand access to the data, this is no longer the case. 
Incentives and disincentives may result in "alternative facts" in the dataset rendering it useless for everybody. Systems need to be designed to have built-in protection for personal and business data, which do not depend on trusting current or future authorities.

All the data domains mentioned as well as the incentives for sharing will be within the scope of the BeeXML standard. The new BeeXML journal is the platform where all the stakeholders can come together to shape the future of bee-related data as a tool for healthy bees and a prosperous beekeeping sector. We invite you to join in and contribute your expertise and ideas to this important effort.

Walter 